LIVER

\title{
Modest weight loss and physical activity in overweight patients with chronic liver disease results in sustained improvements in alanine aminotransferase, fasting insulin, and quality of life
}

\author{
I J Hickman, J R Jonsson, J B Prins, S Ash, D M Purdie, A D Clouston, E E Powell
}

Gut 2004;53:413-419. doi: 10.1136/gut.2003.027581

Background and aim: Obesity is a risk factor for progression of fibrosis in chronic liver diseases such as non-alcoholic fatty liver disease and hepatitis $C$. The aim of this study was to investigate the longer term effect of weight loss on liver biochemistry, serum insulin levels, and quality of life in overweight patients with liver disease and the effect of subsequent weight maintenance or regain.

Patients: Thirty one patients completed a 15 month diet and exercise intervention.

See end of article for authors' affiliations

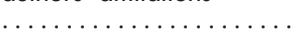

Correspondence to: Dr E Powell, Princess Alexandra Hospital, Ipswich Rd, Woolloongabba, Qld, 4102, Australia; Elizabeth_Powell@ health.qld.gov.au

Accepted for publication 20 October 2003
Results: On completion of the intervention, 21 patients (68\%) had achieved and maintained weight loss with a mean reduction of 9.4 (4.0)\% body weight. Improvements in serum alanine aminotransferase (ALT) levels were correlated with the amount of weight loss $(r=0.35, p=0.04)$. In patients who maintained weight loss, mean ALT levels at 15 months remained significantly lower than values at enrolment $(p=0.004)$, while in regainers $(n=10)$, mean ALT levels at 15 months were no different to values at enrolment $(p=0.79)$. Improvements in fasting serum insulin levels were also correlated with weight loss $(r=0.46, p=0.04)$, and subsequent weight maintenance sustained this improvement. Quality of life was significantly improved after weight loss. Weight maintainers sustained recommended levels of physical activity and had higher fasting insulin levels $(p=0.03)$ at enrolment than weight regainers.

Conclusion: In summary, these findings demonstrate that maintenance of weight loss and exercise in overweight patients with liver disease results in a sustained improvement in liver enzymes, serum insulin levels, and quality of life. Treatment of overweight patients should form an important component of the management of those with chronic liver disease.
$\mathrm{T}$ he prevalence of obesity and overweight has risen at an alarming rate over the past 20 years. ${ }^{1}$ In addition to increasing the risk of the development of type 2 diabetes, hypertension, and dyslipidaemia, excess body weight also has an adverse effect on the liver. Non-alcoholic fatty liver disease (NAFLD) is an increasingly recognised condition that is often seen in patients who are overweight or diabetic or in those with the insulin resistance syndrome. ${ }^{2}$ It encompasses a wide spectrum of liver pathology, ranging from steatosis to steatohepatitis, fibrosis, and cirrhosis. ${ }^{3}$ In patients with NAFLD, the clinical risk factors that are associated with liver injury include elevated body mass index (BMI), visceral adiposity (increased waist circumference), presence of type 2 diabetes, and systemic hypertension. ${ }^{245}$

Obesity is also recognised as an independent risk factor for the progression of fibrosis in other chronic liver diseases. ${ }^{6} \mathrm{~A}$ number of studies have now demonstrated an association between increased BMI or visceral adiposity and hepatic steatosis $^{7}$ and fibrosis ${ }^{8}$ in patients infected with hepatitis $C$ virus (HCV). In overweight patients with chronic HCV, we recently demonstrated an association between increasing insulin levels and increasing hepatic fibrosis, suggesting that host metabolic factors also contribute to disease progression. ${ }^{9}$ Similarly, in patients with alcoholic liver disease, elevated BMI and fasting blood glucose were independent risk factors for hepatic fibrosis. ${ }^{10}$

The role of increased BMI and steatosis as comorbid factors in the progression of fibrosis has important therapeutic implications. Although gradual weight reduction is recommended as a first step in the management of patients with obesity related fatty liver, there are a paucity of long term outcome data on the effect of modest weight loss on liver disease or associated metabolic factors. We have previously reported the early results of a three month weight reduction programme in patients with steatosis associated with chronic HCV. ${ }^{11}$ Modest weight loss in these patients was associated with an improvement in abnormal liver enzymes due to a reduction in steatosis, and in some patients an improvement in necroinflammatory activity and fibrosis. However, the effect of modest weight loss on liver histology and metabolic factors in patients with NAFLD and other chronic liver diseases is less clear. In addition, there are no data on the ability of patients to sustain weight loss long term and the effect of subsequent weight maintenance or regain on liver disease and metabolic parameters.

A number of studies have consistently reported impairment in health related quality of life (HRQL) in patients with chronic liver disease compared with healthy individuals. ${ }^{12-15}$ In addition, there is a dose-response relationship between BMI and the degree of HRQL impairment. ${ }^{16}$ It remains unknown whether the beneficial effects of weight reduction on HRQL are observed in patients with chronic liver disease and are sustainable long term.

Abbreviations: HCV, hepatitis C virus; NAFLD, non-alcoholic fatty liver disease; NASH, non-alcoholic steatohepatitis; BMI, body mass index; HOMA, homeostasis model of assessment; ALT, alanine aminotransferase; HRQL, health related quality of life; SF36, short form 36; PCS, physical component score; MCS, mental component score 
The aim of this study was to investigate the longer term effect of a lifestyle intervention involving weight loss and increased physical activity on liver biochemistry, fasting insulin levels, and HRQL in overweight patients with liver disease. The effect of subsequent weight maintenance or weight regain on these biochemical, metabolic, and quality of life parameters was determined. In addition, we sought to determine indicators for successful maintenance of weight loss.

\section{METHODS}

\section{Patient enrolment and compliance}

Forty three patients with hepatic steatosis seen in the liver clinic at Princess Alexandra Hospital between 1999 and 2000 were invited to participate in the study. Informed consent was obtained from each patient and the study protocol was approved by the hospital research ethics committee. Criteria for entry into the study were liver biopsy demonstrating $\geqslant$ grade 1 steatosis, overweight or obese (BMI $\geqslant 25 \mathrm{~kg} / \mathrm{m}^{2}$ in Caucasians and $\geqslant 23 \mathrm{~kg} / \mathrm{m}^{2}$ in Asians) or weight gain of $>10 \%$ of usual body weight within 12 months, and alcohol consumption $<10 \mathrm{~g} /$ day.

Twenty seven patients had hepatic steatosis in association with chronic hepatitis $\mathrm{C}$ and 11 of these patients were included in an earlier report describing the initial response to a three month weight reduction programme. ${ }^{11}$ Data from these patients were included in this study to monitor longer term outcomes. All patients with chronic HCV were ineligible for, or non-responders to, current antiviral treatment. Sixteen patients with non-HCV obesity related steatosis were included. Ten of these 16 patients had a clinical and histological diagnosis of NAFLD. In another four non-HCV patients, steatosis was present in association with another non-steatogenic primary liver disease (inactive hepatitis $\mathrm{B}$, $\mathrm{n}=2$; nitrofurantoin induced autoimmune chronic active hepatitis, $\mathrm{n}=1$; and primary biliary cirrhosis, $\mathrm{n}=1$ ). In the remaining two patients, one had been treated for pituitary Cushing's disease and another had previously received tamoxifen therapy for breast cancer which had been ceased for six months. This patient cohort reflects the increasingly common finding of overweight and steatosis in association with another liver disease. When steatosis was present with hepatocyte ballooning, Mallory's hyaline or subsinusoidal fibrosis in the acini, patients were subclassified as nonalcoholic steatohepatitis (NASH) ${ }^{17}$

Eight patients withdrew during the initial three month weight reduction period ( $\mathrm{HCV}, \mathrm{n}=6$; non- $\mathrm{HCV}, \mathrm{n}=2$ ) and a further four patients withdrew during the 12 month weight maintenance period $(\mathrm{HCV}, \mathrm{n}=3$; non- $\mathrm{HCV}, \mathrm{n}=1)$. The reasons for withdrawal were non-compliance with the programme protocol $(n=6)$, alcohol intake in excess of study exclusion criteria (20 g/day and $45 \mathrm{~g} /$ day, $\mathrm{n}=2$ ), work commitments $(n=2)$, and psychiatric disorders preventing attendance at clinic $(n=2)$. Non-compliance was considered if a patient missed two consecutive appointments and was unable to be contacted via telephone or mail. Overall, 31 patients ( $\mathrm{HCV}, \mathrm{n}=18$; non-HCV $\mathrm{n}=13$ ) completed the entire 15 month programme (72\% completion rate).

\section{Lifestyle intervention}

The 15 month lifestyle intervention was divided into two periods: an initial three month weight reduction period $(\mathrm{t}=0$ to $\mathrm{t}=3$ months) followed by a 12 month weight maintenance period $(t=4$ to $t=15$ months). During the initial three month period, patients were seen on a weekly basis by a dietician, as previously described. ${ }^{11}$ Physical activity was recommended at 150 minutes of aerobic exercise each week. During the subsequent 12 month follow up period, patients were seen on a monthly basis by a dietician, and encouraged to maintain an appropriate diet and exercise routine.

Weight and waist circumference measurements and details on alcohol, exercise, and diet were recorded at each visit. The macronutrient content of the diets was analysed using Foodworks version 2.10.133 (Xyris Software 1998-2000, Australia). No vitamin, mineral, or herbal preparations were allowed. Weight maintenance at $\mathrm{t}=15$ months was defined as weight regain of $<25 \%$ of initial weight loss. ${ }^{18}$

\section{Biochemical and metabolic studies}

Blood samples for measurement of liver enzymes, cholesterol, triglycerides, glucose, and insulin were obtained after an overnight fast at months 0,3 , and 15. Routine biochemical tests were performed using a Hitachi 747-100 analyser (Roche, Australia). Circulating insulin was determined using the Tosoh AIA600 analyser two site immunoenzymometric assay (Tosoh Medics, San Francisco, California, USA) with a coefficient of variation of $4-5 \%$. Insulin resistance was determined using the homeostasis model of assessment $(\text { HOMA })^{19}$ with an upper threshold of $1.64 .^{20}$

\section{Liver histology}

Liver biopsy specimens were fixed in 10\% neutral buffered formalin and embedded in paraffin. Steatosis was graded as follows: $0 \quad(<5 \%$ of hepatocytes affected $) ; 1 \quad(5-30 \%$ of hepatocytes affected); 2 (30-70\% of hepatocytes affected); or $3(>70 \%$ of hepatocytes affected). The degree of inflammation and fibrosis was assessed and graded using the modified Knodell scoring system of Ishak. ${ }^{21}$ Sections were analysed by an experienced hepatopathologist (AC) who was blinded to patient identity and time of biopsy.

\section{Health related quality of life (HRQL)}

HRQL was measured at months 0,3 , and 15 using the short form 36 (SF-36) questionnaire. ${ }^{22}$ The SF-36 questionnaire measured eight multi-item scales called health domains (physical functioning, physical role limitation, bodily pain, general health, vitality, social functioning, emotional role limitation, and mental health). Scores were assembled and transformed using previously described methods. ${ }^{22}$ Higher transformed scores indicated better health. Two summary scores, the mental component score (MCS) and the physical component score (PCS), were calculated via a weighted combination of the eight health domains. SF-36 scores obtained from patients with chronic liver disease were compared with Australian population norms. ${ }^{23}$

\section{Statistical analysis}

Continuous normally distributed variables were summarised as mean (SD) (mean (SEM) for graphical representation). Alcohol intake, degree of steatosis, and stage of fibrosis all being either skewed or ordinal were summarised using the median. The degree of association between continuous normally distributed variables was assessed using Pearson's correlation coefficient $(r)$. The degree of association between any ordinal or non-normal variables was measured using Spearman's non-parametric correlation coefficient $\left(r_{\mathrm{s}}\right)$.

Changes in weight, waist circumference, and dietary intake were assessed using paired $t$ tests. Changes in the degree of steatosis, stage of fibrosis, or grade of inflammation over the intervention period were assessed using the Wilcoxon signed rank test. Differences in mean anthropometric, biochemical, and metabolic factors between patients who maintained or regained weight were assessed using the Wilcoxon rank sum test. Categorical data were compared using a $\chi^{2}$ test. All analysis was carried out using SPSS software version 10.0 (SPSS Inc. Chicago, Illinois, USA). Statistical significance was taken at a level of $5 \%$. 


\section{RESULTS}

\section{Study patients}

Demographic information for the 35 patients (HCV $\mathrm{n}=21$; non-HCV, $\mathrm{n}=14$ ) who completed the three month weight reduction programme and entered the 12 month weight maintenance programme is summarised in table 1. Only one patient did not have an elevated BMI $\left(21 \mathrm{~kg} / \mathrm{m}^{2}\right)$ but she had HCV genotype 3 and had experienced a recent weight gain of $>10 \mathrm{~kg}$ over the prior 12 months. BMI of all patients ranged from 21.2 to $51.4 \mathrm{~kg} / \mathrm{m}^{2}$. Median alcohol intake was $0 \mathrm{~g} /$ day (range $0-7 \mathrm{~g} /$ day). Three patients (HCV, $\mathrm{n}=1$; non-HCV, $\mathrm{n}=2$ ) had type 2 diabetes according to defined criteria. ${ }^{24}$ At entry, 25 of 35 patients had grade 2 or 3 steatosis, and additional histological features of NASH were present in eight patients ( $\mathrm{HCV}, \mathrm{n}=2 v$ non-HCV, $\mathrm{n}=6 ; \mathrm{p}=0.04)$. Ten patients had moderate to severe fibrosis (Knodell fibrosis score $\geqslant 4$ ), including three patients with cirrhosis (HCV, $\mathrm{n}=2$; non-HCV, $\mathrm{n}=1$ ). Of 21 patients with chronic HCV entering the maintenance programme, 10 patients had viral genotype 1 , one patient had viral genotype 2 , and 10 patients had viral genotype 3.

\section{Response to the 15 month lifestyle intervention: compliance}

At $\mathrm{t}=3$ months there was a mean weight loss of $5.8(3.4) \%$ body weight and a mean decrease in waist circumference of $9.1(5.4) \mathrm{cm}$. At $\mathrm{t}=15$ months, 21 (68\%) patients (HCV, $\mathrm{n}=11$; non-HCV, $\mathrm{n}=10$ ) had maintained weight loss according to defined criteria ${ }^{18}$ with a total mean weight loss of $9.4(4.0) \%$ body weight and decrease in waist circumference of $13.0(5.0) \mathrm{cm}$ (fig 1A, B). Six patients normalised $\mathrm{BMI}$ at $\mathrm{t}=15$ months ( $\mathrm{HCV}, \mathrm{n}=4$; non-HCV, $\mathrm{n}=2$ ). Ten $(32 \%)$ patients ( $\mathrm{HCV}, \mathrm{n}=7$; non-HCV, $\mathrm{n}=3$ ) had regained weight. In this latter group there was a mean increase in weight of $8.6(5.3) \%$ body weight and a mean increase in waist circumference of $5.4(3.0) \mathrm{cm}$ relative to the end of the three month intensive period (fig $1 \mathrm{~A}, \mathrm{~B}$ ). Weight at $\mathrm{t}=15$ months was no different from $\mathrm{t}=0$ in the weight regainers $(p=0.71)$ although waist circumference remained significantly lower than enrolment measurements $(\mathrm{p}=0.002)$ (fig lB). There was no difference in weight and waist change between HCV and non-HCV patients.

\section{Diet and exercise}

Diet diaries at $t=0$ and $t=3$ months revealed a $43 \%$ decrease in energy intake $(\mathrm{p}<0.0001)$ and a $52 \%$ reduction in total fat
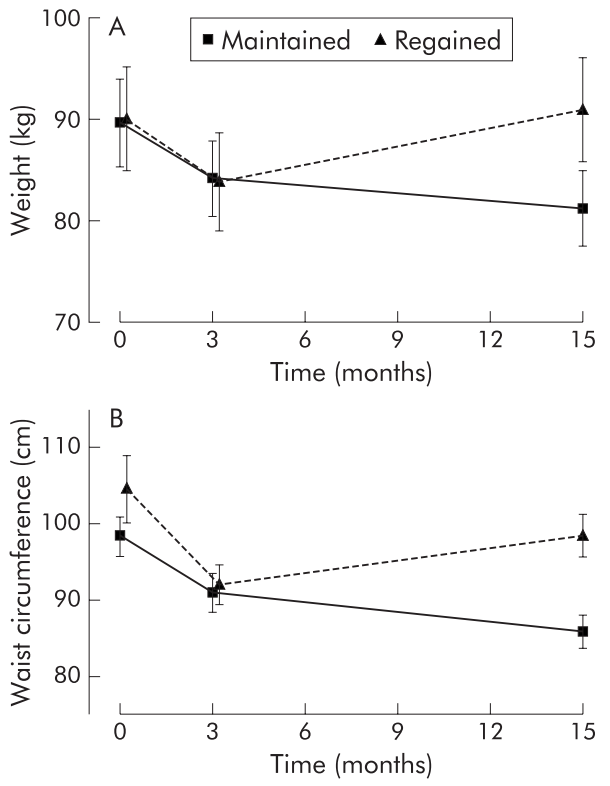

Figure 1 Change in mean (SEM) weight ( $A$ ) and mean (SEM) waist circumference (B) during the 15 month lifestyle programme in patients who maintained and regained weight. Intensive period ( $t=0$ to $t=3$ months), $n=35$; and after subsequent weight maintenance $(n=21)$ and weight regain $(n=10)$, during the 12 month period $(t=3$ to $t=15$ months).

content $(\mathrm{p}<0.0001)$, and energy from saturated fat decreased from $13(1) \%$ to 10 (1)\% of total energy intake per day $(p=0.005)$. The decrease in energy intake reported would be expected to result in greater weight losses than were observed (approximately $1 \mathrm{~kg} /$ week). Systematic underreporting of dietary intake is common in overweight patients and should be considered when assessing dietary composition. At enrolment there was no difference in dietary macronutrient composition between patients with $\mathrm{HCV}$ and non-HCV related liver disease. There was no association between macronutrient composition and insulin resistance.

Exercise diaries were not available for two patients. Before commencement of the programme only 10 patients $(29 \%)$ were involved in any form of regular exercise (range 40$350 \mathrm{~min} /$ week). From $\mathrm{t}=0$ to $\mathrm{t}=3$ months, all patients

Table 1 Demographic, biochemical, metabolic, and histological parameters of patients in the lifestyle intervention programme

\begin{tabular}{|c|c|c|c|c|c|}
\hline Parameter & $\begin{array}{l}\text { Total group } \\
(\mathrm{n}=35)\end{array}$ & $\begin{array}{l}\mathrm{HCV} \\
(n=21)\end{array}$ & $\begin{array}{l}\text { Non-HCV } \\
(n=14)\end{array}$ & p Value* & Reference range \\
\hline Age (y) & $43.8(9.6)$ & $43.4(9.8)$ & 44.4 (9.7) & 0.77 & \\
\hline Sex M/F† (\% male) & $21 / 14(60)$ & $11 / 10(52)$ & $10 / 4(71)$ & 0.26 & \\
\hline Type 2 DM (n) & 3 & 1 & 2 & & \\
\hline BMI $\left(\mathrm{kg} / \mathrm{m}^{2}\right)$ & $30.5(5.9)$ & $29.3(4.5)$ & $32.3(7.3)$ & 0.14 & \\
\hline Waist $(\mathrm{cm})$ & $100(12)$ & $99(11)$ & $103(14)$ & 0.26 & \\
\hline ALT (U/I) & $113(78)$ & $138(85)$ & $76(49)$ & 0.02 & $<35 \mathrm{U} / \mathrm{I}$ \\
\hline Cholesterol (mmol/l) & $4.3(1.3)$ & $3.7(1.2)$ & $5.3(0.8)$ & $<0.0001$ & $<5.5 \mathrm{mmol} / \mathrm{l}$ \\
\hline Triglyceride (mmol/l) & $1.4(1.0)$ & $1.2(0.8)$ & $1.8(1.2)$ & 0.12 & $<2.0 \mathrm{mmol} / \mathrm{l}$ \\
\hline Insulin (mU/I) & $15(7)$ & $14(7)$ & $15(7)$ & 0.86 & $<20 \mathrm{mU} / \mathrm{l}$ \\
\hline Glucose (mmol/l) & 5.7 (1.4) & $5.3(1.3)$ & 6.1 (1.4) & 0.09 & $<6.0 \mathrm{mmol} / \mathrm{l}$ \\
\hline HOMA & 3.7 (2.6) & $3.7(3.0)$ & 3.7 (1.8) & 0.99 & $<1.64$ \\
\hline Steatosis (median) & 2 & 2 & 2 & 0.15 & \\
\hline $\mathrm{NASH}+(\mathrm{n})$ & 8 & 2 & 6 & 0.04 & \\
\hline $\begin{array}{l}\text { Fibrosis (median) } \\
\text { (Knodell score 0-6) }\end{array}$ & 2 & 3 & 1 & 0.09 & \\
\hline
\end{tabular}

Values are mean (SD) or number.

*Independent $t$ test HCV versus non-HCV groups; †Fisher's exact test.

$\mathrm{HCV}$, hepatitis C virus; NASH, non-alcoholic steatohepatitis; $\mathrm{BMI}$, body mass index; HOMA, homeostasis model of assessment; ALT, alanine aminotransferase; DM, diabetes mellitus. 
except two (6\%) commenced regular weekly aerobic activity with mean exercise time of 214 (166) minutes per week (range $0-840 \mathrm{~min} /$ week). During the $12 \mathrm{month}$ weight maintenance programme, overall exercise decreased to a mean of 120 (140) min/week (range 0-560 min/week). In those patients who maintained weight, exercise levels were sustained at recommended levels of 150 (160) min/week whereas those that regained weight had reduced their level of exercise to $50(54) \mathrm{min} /$ week $(\mathrm{p}=0.02)$.

\section{Biochemical and metabolic changes during the lifestyle intervention}

Figure 2 represents the per cent of initial serum alanine aminotransferase (ALT) levels in patients who maintained and regained weight during the lifestyle intervention. From $\mathrm{t}=0$ to $\mathrm{t}=3$ months, serum ALT levels improved significantly with weight reduction $(\mathrm{p}=0.001)$. There was a mean reduction in ALT of $17 \%$ (133 (91) U/l to 100 (66) U/l; $\mathrm{p}=0.02)$ and $26 \%(77$ (42) U/l to $48(20) \mathrm{U} / \mathrm{l} ; \mathrm{p}=0.02)$ in HCV and non-HCV groups, respectively. Patients with viral genotype 3 had a significantly greater improvement in ALT than those with viral genotype $l(p=0.008)$. The decrease in serum ALT levels was associated with the amount of weight loss $(r=0.35, \mathrm{p}=0.04)$.

In patients who maintained their weight loss at $t=15$, mean serum ALT levels remained significantly lower than ALT levels at $\mathrm{t}=0(\mathrm{p}=0.004)$ and 11 patients had normalised serum ALT levels ( $\mathrm{HCV} n=2$; non-HCV, $n=9$ ). In contrast, in patients who regained weight, mean serum ALT levels rose during the maintenance programme and at $t=15$ were no different to values at $\mathrm{t}=0(\mathrm{p}=0.79)$ (fig 2).

In addition to decreasing serum ALT levels, weight reduction significantly decreased fasting insulin levels, and subsequent weight maintenance resulted in a sustained improvement $(\mathrm{p}=0.03)$ (fig 3$)$. In patients who regained weight, there was no significant change in fasting insulin levels between $\mathrm{t}=0$ and $\mathrm{t}=15$ months $(\mathrm{p}=0.75)$ irrespective of the amount of exercise reported during the intervention. The amount of weight loss correlated with the reduction in fasting serum insulin levels $(r=0.46, \mathrm{p}=0.035)$ but not with the change in HOMA score $(p=0.72)$. Despite similar changes in weight, patients with HCV had a significantly greater decrease in fasting insulin during the initial three month period compared with non-HCV patients $(p=0.01)$ but there was no difference between groups at 15 months $(\mathrm{p}=0.61)$.

\section{Health related quality of life}

At enrolment, BMI had a strong negative correlation with the HRQL physical component score $\left(r_{\mathrm{s}}=-0.48, \mathrm{p}=0.004\right)$ and was also negatively correlated with four SF-36 health domains, including physical functioning $(r=-0.54$,

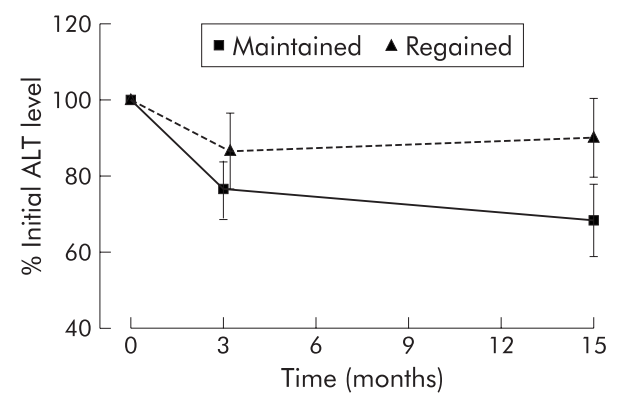

Figure 2 Mean (SEM) per cent of initial serum alanine aminotransferase (ALT) levels during the lifestyle intervention at 3 and 15 months in patients who maintained $(n=21)$ and regained $(n=10)$ weight.

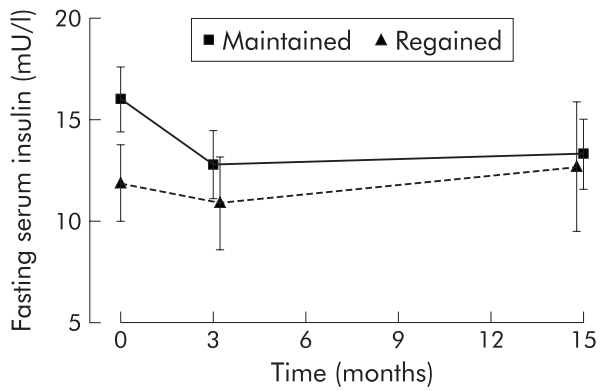

Figure 3 Change in mean (SEM) fasting serum insulin levels during the lifestyle intervention at 3 and 15 months in patients who maintained $(n=16)$ and regained $(n=7)$ weight.

$\mathrm{p}=0.001)$, general health $(r=-0.40, \mathrm{p}=0.02)$, social functioning $(r=-0.40, \mathrm{p}=0.02)$, and bodily pain $(r=-0.40, \mathrm{p}=0.03)$. Compared with population norms, ${ }^{23}$ both the PCS and MCS were significantly decreased ( $p=0.0003$ and $p=0.0007$, respectively) (fig $4 A, B$ ) and seven of the eight SF-36 health domains scored significantly lower in patients with chronic liver disease at $t=0$. After the initial three month intervention, PCS and MCS significantly increased $(p<0.0001$ and $p=0.004$, respectively) (fig 4A, B) and all but one health domain were comparable with population norms. In patients who maintained weight at $\mathrm{t}=15$, both PCS and MCS remained significantly higher than enrolment scores $(p=0.005$ and $p=0.003$, respectively). In contrast, in patients who regained weight, PCS and MCS scores decreased after 15 months and were no different to those at enrolment $(p=0.12$ and $p=0.06$, respectively)
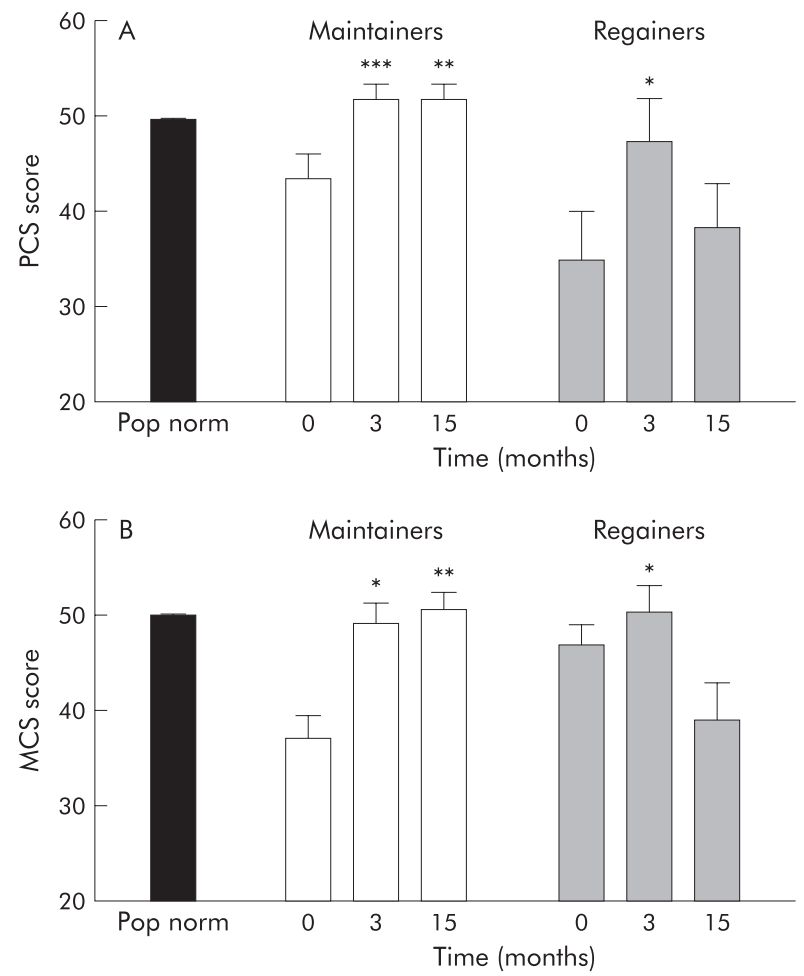

Figure 4 Physical component scores (PCS (A)) and mental component scores (MCS (B)) for patients who maintained $(n=15)$ and regained $(n=10)$ weight, before $(t=0)$ and after $(t=3)$ the initial three month intervention and after 15 months of follow up. Pop norm, Australian population norms. Compared with $t=0$ values, ${ }^{*} p<0.05,{ }^{* *} p<0.01$, and ${ }^{* * *} p<0.0001$. 
(fig 4A, B). Although mean PCS score was higher at $\mathrm{t}=0$ in patients who maintained weight, this did not reach statistical significance $(p=0.10)$. There was no association between fibrosis score and quality of life in patients with chronic liver disease.

\section{Factors associated with successful weight maintenance}

The amount of physical activity per week played a significant role in the overall success of weight loss and maintenance. Patients who maintained weight loss were more likely to have attained realistic levels of exercise in accordance with recommendations and continued exercising long term $(p=0.02)$. Weight regainers attempted significantly higher levels of exercise during the initial three month weight loss period $(p=0.04)$, which was reflected in the increased loss of waist circumference at three months $(p=0.02)$ (table 2). However, this was not sustained long term.

Insulin resistance did not impact on a patient's ability to decrease weight or waist circumference during the initial three month period. However, the ability to maintain this weight loss for 12 months was significantly associated with insulin resistance. Weight maintainers had significantly higher fasting insulin levels $(p=0.03)$ and HOMA $(\mathrm{p}=0.02)$ at $\mathrm{t}=0$ than those patients who regained weight. There was a significant negative correlation between the amount of weight regained during follow up and metabolic factors associated with insulin resistance such as fasting insulin $\left(r_{\mathrm{s}}=-0.47, \mathrm{p}=0.01\right)$, glucose $\left(r_{\mathrm{s}}=-0.40, \mathrm{p}=0.03\right)$, and HOMA $\left(r_{\mathrm{s}}=-0.54, \mathrm{p}=0.002\right)$.

No other patient or disease characteristic was associated with successful weight maintenance (table 2).

\section{Histology}

Fourteen patients consented to a repeat biopsy 3-6 months after completion of the initial three month period. These repeat biopsies were offered to monitor the short term effects of weight reduction on liver histology and some of the data have been included in an earlier report. ${ }^{11}$ Further biopsies after 15 months of the programme were not considered clinically indicated or ethically justified, especially in those patients with a sustained improvement in liver enzymes. Overall, there was a striking improvement in steatosis after weight reduction $(p<0.0001)$ (fig 5$)$. In seven patients there was also an improvement in the stage of fibrosis $(p=0.02)$ (fig 6).

In patients with an improvement in fibrosis score after weight loss, fasting insulin levels at enrolment were significantly higher compared with those whose fibrosis

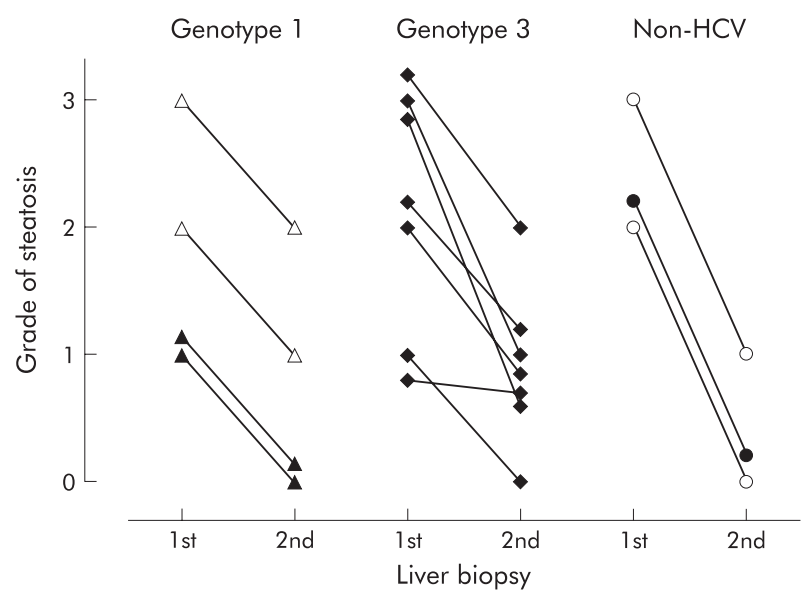

Figure 5 Grade of hepatic steatosis in patients before (1 st biopsy) and after (2nd biopsy) weight reduction. Hepatitis $\mathrm{C}$ virus (HCV) genotype 1 $(n=4) ; \mathrm{HCV}$ genotype $3(n=7)$; and non-HCV $(n=3)$. Total group median before $=2$ and after $=1(p<0.0001)$. Open symbols represent those patients with additional histological features of non-alcoholic steatohepatitis.

score did not improve (16 (6) v ll (4) mU/l, respectively; $\mathrm{p}=0.02)$. In addition, there was greater improvement in ALT levels at three months in those patients whose fibrosis score improved compared with those with no improvement $(\mathrm{p}=0.03)$.

\section{DISCUSSION}

These results demonstrate that maintenance of weight reduction and increased physical activity result in a sustained improvement in ALT, fasting insulin levels, and HRQL in overweight patients with chronic liver disease. This sustained improvement was seen both in patients with NAFLD and in those with steatosis in association with another chronic liver disease.

In an earlier study, we demonstrated that in the short term, weight loss reduced hepatic steatosis and fibrosis in patients with chronic HCV. ${ }^{11}$ In the current study, we demonstrated a similar early histological improvement in an additional small number of patients with obesity related fatty liver disease. Although liver biopsies were not performed at 15 months, it is likely that the sustained improvement in ALT and fasting insulin in patients who maintained weight loss was accompanied by a sustained reduction in hepatic steatosis and necroinflammatory

Table 2 Factors associated with long term weight loss and weight maintenance or regain after a lifestyle intervention

\begin{tabular}{|c|c|c|c|}
\hline Factors at onset or during lifestyle intervention & $\begin{array}{l}\text { Maintainers } \\
(n=21)\end{array}$ & $\begin{array}{l}\text { Regainers } \\
(\mathrm{n}=10)\end{array}$ & p Value \\
\hline Age (y) & $43.1(8.0)$ & $41.5(10.3)$ & 0.64 \\
\hline $\operatorname{Sex}(M / F)^{*}(\%$ male $)$ & $14 / 7(67)$ & $7 / 3(70)$ & 0.85 \\
\hline Initial BMI $\left(\mathrm{kg} / \mathrm{m}^{2}\right)$ & $30.6(6.6)$ & $30.6(4.4)$ & 0.98 \\
\hline Weight loss $t=0$ to $t=3(\%)$ & 5.9 (3.3) & $6.8(3.2)$ & 0.47 \\
\hline Waist loss $t=0$ to $t=3(\%)$ & $7.5(4.0)$ & $11.6(4.9)$ & 0.02 \\
\hline $\mathrm{ALT} \mathrm{t}=0(\mathrm{U} / \mathrm{I})$ & $107(75)$ & $115(89)$ & 0.80 \\
\hline Insulin $t=0$ (mU/l) & $16(6)$ & $11(5)^{\circ}$ & 0.03 \\
\hline $\mathrm{HOMA} t=0$ & $4.1(1.7)$ & $2.5(1.3)$ & 0.02 \\
\hline Non-HCV/HCV (\%HCV) & $10 / 11(52)$ & $3 / 7(70)$ & 0.35 \\
\hline Amount exercise $t=0$ to $t=3$ ( $\mathrm{min} /$ week) & 185 (195) & $245(82)$ & 0.04 \\
\hline Amount of exercise $t=3$ to $t=15$ ( $\mathrm{min} /$ week) & $150(160)$ & $50(55)$ & 0.02 \\
\hline
\end{tabular}

${ }^{*} \chi^{2}$.

Values are mean (SD) or number

$\mathrm{HCV}$, hepatitis $\mathrm{C}$ virus; $\mathrm{BMI}$, body mass index; HOMA, homeostasis model of assessment; $\mathrm{ALT}$, alanine aminotransferase. 


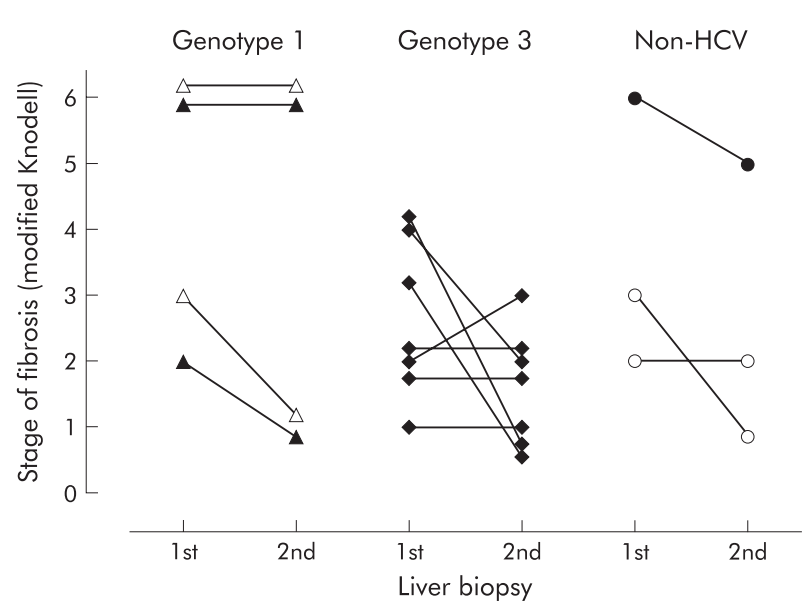

Figure 6 Modified Knodell fibrosis score in patients before (1 st biopsy) and after (2nd biopsy) weight reduction. Hepatitis $\mathrm{C}$ virus (HCV) genotype $1(n=4) ; \mathrm{HCV}$ genotype $3(n=7)$; and non-HCV $(n=3)$. Total group median before $=3$ and after $=2(p=0.02)$. Open symbols represent those patients with additional histological features of nonalcoholic steatohepatitis.

activity. With long term weight maintenance there is likely to be an even greater resolution of hepatic fibrosis.

Overall, in our patient cohort, the decrease in ALT and insulin levels was associated with the amount of weight loss. However, a sustained improvement in ALT and insulin levels was seen with a weight loss of as little as $4-5 \%$ body weight without necessarily normalising BMI. These findings are in accordance with results of recent type 2 diabetes intervention studies where the average amount of weight loss was not large yet resulted in a substantial reduction in the incidence of diabetes..$^{25-27}$ Without intervention, an average population weight gain of $>1.5 \mathrm{~kg} /$ year could be expected..$^{28}$ Completion of this intervention prevented expected annual weight gain for $84 \%$ of patients and maintained a significant weight reduction in $68 \%$ of patients. Waist circumference remained significantly below enrolment measurements in all but one patient, regardless of weight change during follow up. Six of 10 patients who regained weight reported continuing low levels of physical activity which may have contributed to a change in body fat distribution despite weight regain.

Our study was not designed to test the relative contribution of dietary changes, weight loss, or increased physical activity to the improvement in liver enzymes and insulin levels, and the individual effects of these components warrant further study.

One of the most important factors associated with successful weight maintenance was the continuation of appropriate levels of physical activity in the long term. Patients who regained weight had significantly higher than recommended exercise levels during the initial three months, suggesting that patients attempting unrealistic short term changes do not sustain these changes and may be more likely to relapse long term. Patients who maintained weight sustained recommended levels of activity during follow up. It is widely accepted that exercise has an important role in the treatment of visceral adiposity and insulin resistance. Our data further support the important role of exercise in the successful maintenance of weight loss in patients with chronic liver disease.

The other factor significantly associated with maintenance of weight loss was insulin resistance. Weight regain was inversely associated with insulin resistance-that is, less regain was observed in patients with higher HOMA and fasting insulin levels. Although controversial, a number of studies in different population groups have shown that hyperinsulinaemia predicts a reduced weight gain over time and may be an adaptation for weight maintenance. ${ }^{29}{ }^{30}$ The mechanisms linking the association between insulin resistance and weight gain remain to be determined but this factor may be useful for predicting those patients at higher risk of weight regain after lifestyle interventions.

The success of weight maintenance in our study was probably due to the initial intensive programme combined with long term follow up. Increasing, the length and frequency of standard dietetic intervention improves long term success. ${ }^{31}$ In addition to the substantial cost of chronic liver disease to the health care system, the reduced HRQL in our patients illustrates the significant personal and social burden on those afflicted. Comorbid conditions such as obesity significantly contribute to the reduced feeling of well being in these patients, irrespective of disease severity. This study demonstrates that investment in weight reduction has the ability to reduce risk factors associated with progression of liver disease, decrease abnormal liver enzymes, improve quality of life, and in a proportion of patients improve histological features of liver injury. Importantly, these changes were achievable and sustainable with relatively small but persistent changes in lifestyle. These results suggest that treatment of overweight patients should form an important component of management of those with chronic liver disease.

\section{ACKNOWLEDGEMENTS}

Funding for this research was provided by the National Health and Medical Research Council (Australia) (IH and EP), Gastroenterology Society of Australia Foundation/Pharmacia and Upjohn Research Fellowship (JJ), and the Princess Alexandra Hospital Research and Development Foundation.

\section{Authors' affiliations}

I J Hickman, School of Medicine, University of Queensland, and Department of Nutrition and Dietetic Services, Princess Alexandra Hospital, Brisbane, Australia

J R Jonsson, A D Clouston, School of Medicine, University of Queensland, Brisbane, Australia

J B Prins, Department of Endocrinology and Diabetes, Princess Alexandra Hospital, Brisbane, Australia

S Ash, Nutrition and Dietetic Services, Princess Alexandra Hospital, Brisbane, Australia

D M Purdie, Population and Clinical Sciences Division, Queensland Institute of Medical Research, Brisbane, Australia

E E Powell, School of Medicine, University of Queensland, and Department of Gastroenterology and Hepatology, Princess Alexandra Hospital, Brisbane, Australia

\section{REFERENCES}

1 Mokdad AH, Ford ES, Bowman BA, et al. Prevalence of obesity, diabetes, and obesity-related health risk factors, 2001. JAMA 2003;289:76-9.

2 Marchesini G, Brizi M, Bianchi G, et al. Nonalcoholic fatty liver disease: a feature of the metabolic syndrome. Diabetes 2001;50:1844-50.

3 McCullough A. Update on nonalcoholic fatty liver disease. J Clin Gastroenterol 2002;34:255-62.

4 Angulo P, Keach JC, Batts KP, et al. Independent predictors of liver fibrosis in patients with nonalcoholic steatohepatitis. Hepatology 1999;30:1356-62.

5 Nakao K, Nakata K, Ohtsubo N, et al. Association between nonalcoholic fatty liver, markers of obesity, and serum leptin level in young adults. Am J Gastroenterol 2002;97:1796-801.

6 Clouston AD, Powell EE. Interaction of non-alcoholic fatty liver disease with other liver diseases. Best Pract Res Clin Gastroenterol 2002;16:767-81.

7 Adinolfi LE, Gambardella M, Andreana A, et al. Steatosis accelerates the progression of liver damage of chronic hepatitis $C$ patients and correlates with specific HCV genotype and visceral obesity. Hepatology 2001;33:1358-64.

8 Hourigan LF, Macdonald GA, Purdie D, et al. Fibrosis in chronic hepatitis C correlates significantly with body mass index and steatosis. Hepatology 1999;29:1215-19

9 Hickman IJ, Powell EE, Prins JB, et al. In overweight patients with chronic hepatitis $C$, circulating insulin is associated with hepatic fibrosis: implications for therapy. J Hepatol 2003;39:1042-8. 
10 Raynard B, Balian A, Fallik D, et al. Risk factors of fibrosis in alcohol-induced liver disease. Hepatology 2002;35:635-8.

11 Hickman IJ, Clouston AD, Macdonald GA, et al. Effect of weight reduction on liver histology and biochemistry in patients with chronic hepatitis C. Gut 2002;51:89-94.

12 Bonkovsky HL, Woolley JM. Reduction of health-related quality of life in chronic hepatitis $\mathrm{C}$ and improvement with interferon therapy. The Consensus Interferon Study Group. Hepatology 1999;29:264-70.

13 Foster GR, Goldin RD, Thomas HC. Chronic hepatitis $\mathrm{C}$ virus infection causes a significant reduction in quality of life in the absence of cirrhosis. Hepatology 1998;27:209-12

14 Ware JE jr, Bayliss MS, Mannocchia M, et al. Health-related quality of life in chronic hepatitis $C$ : impact of disease and treatment response. The Interventional Therapy Group. Hepatology 1999;30:550-5.

15 Hussain KB, Fontana RJ, Moyer CA, et al. Comorbid illness is an important determinant of health-related quality of life in patients with chronic hepatitis $C$. Am J Gastroenterol 2001;96:2737-44.

16 Fontaine KR, Barofsky I. Obesity and health-related quality of life. Obes Rev $2001 ; 2: 173-82$.

17 Matteoni CA, Younossi ZM, Gramlich T, et al. Nonalcoholic fatty liver disease: a spectrum of clinical and pathological severity. Gastroenterology 1999;116:1413-19.

18 Hill JO, Hauptman J, Anderson JW, etal. Orlistat, a lipase inhibitor, for weight maintenance after conventional dieting: a 1 -y study. Am J Clin Nutr 1999;69:1 108-16.

19 Matthews DR, Hosker JP, Rudenski AS, et al. Homeostasis model assessment: insulin resistance and beta-cell function from fasting plasma glucose and insulin concentrations in man. Diabetologia 1985;28:412-19.

20 Chitturi S, Abeygunasekera S, Farrell G, et al. NASH and insulin resistance: Insulin hypersecretion and specific association with the insulin resistance syndrome. Hepatology 2002;35:373-9.

21 Knodell RG, Ishak KG, Black WC, et al. Formulation and application of a numerical scoring system for assessing histological activity in asymptomatic chronic active hepatitis. Hepatology 1981;1:431-5.
22 Ware JE ir, Sherbourne CD. The MOS 36-item short-form health survey (SF36). I. Conceptual framework and item selection. Med Care 1992;30:473-83.

23 Australian Bureau of Statistics. National Health Survey: SF-36 Population Norms. Canberra, Australia: Australian Bureau of Statistics, 1995.

24 World Health Organisation. Definition, diagnosis and classification of diabetes mellitus and its complications: Report of a WHO consultation. Part 1: Diagnosis and classification of diabetes mellitus. Geneva: World Health Org, 1999 (available from http://whqlibdoc.who.int/hq/1999/ WHO_NCD_NCS_99.2.pdf, 1999).

25 Tuomilehto J, Lindstrom J, Eriksson JG, et al. Prevention of type 2 diabetes mellitus by changes in lifestyle among subjects with impaired glucose tolerance. N Engl J Med 2001;344:1343-50.

26 Pan XR, Li GW, Hu YH, et al. Effects of diet and exercise in preventing NIDDM in people with impaired glucose tolerance. The Da Qing IGT and Diabetes Study. Diabetes Care 1997;20:537-44.

27 Knowler WC, Barrett-Connor E, Fowler SE, et al. Reduction in the incidence of type 2 diabetes with lifestyle intervention or metformin. N Engl J Med 2002;346:393-403.

28 Rothacker DQ, Blackburn GL. Obesity prevalence by age group and 5-year changes in adults residing in rural Wisconsin. J Am Diet Assoc 2000;100:784-90.

29 Schwartz M, Boyko E, Kahn S, et al. Reduced insulin secretion: an independent predictor of body weight gain. J Clin Endocrinol Metab 1995;80:1571-6.

30 Hoag S, Marshall JA, Jones RH, et al. High fasting insulin levels associated with lower rates of weight gain in persons with normal glucose tolerance: the San Luis Valley Diabetes Study. Int J Obes Relat Metab Disord 1995; 19:175-80.

31 Perri MG, Nezu AM, Patti ET, et al. Effect of length of treatment on weight loss. J Consult Clin Psychol 1989;57:450-2. 